\title{
Synthesis of pyrrolo[3,2-a]phenazines from 5-nitroindoles and anilines
}

\author{
Zbigniew Wróbel $\cdot$ Michał Więcław $\cdot$ \\ Robert Bujok $\cdot$ Krzysztof Wojciechowski
}

Received: 25 April 2013/Accepted: 10 September 2013/Published online: 10 October 2013

(C) The Author(s) 2013. This article is published with open access at Springerlink.com

\begin{abstract}
Anilines react with 5-nitroindoles in the presence of $t$-BuOK in $N, N$-dimethylformamide (DMF) to form 5-nitroso-4-arylaminoindoles that in turn when treated with $\mathrm{N}, \mathrm{O}$-bis(trimethylsilyl)acetamide cyclize to pyrrolo[3,2- $a]$ phenazines. In an alternative approach pyrrolo[3,2- $a$ ]phenazines are formed from aminoindoles and nitroarenes.
\end{abstract}

Keywords Amines - Anions · Heterocycles ·

Cyclizations · Nucleophilic substitutions · Lewis acids

\section{Introduction}

Phenazine derivatives are an important class of condensed heterocycles of natural origin [1-4]. Selected methods of synthesizing the phenazine framework are presented in Scheme 1. One of the oldest methods is the reaction of anilines with nitroarenes under basic conditions (the WohlAue reaction, path a) [5]. The Holliman synthesis of phenazines (path b) is a base-induced cyclization of orthonitrodiphenylamines [6]. In the Bamberger-Ham reaction (path c) nitrosobenzenes dimerize under acidic conditions

Z. Wróbel · R. Bujok · K. Wojciechowski ( $\square)$ Institute of Organic Chemistry, Polish Academy of Sciences, ul. Kasprzaka 44/52, P.O. Box 58, 01-224 Warsaw, Poland e-mail: kris@icho.edu.pl

M. Więcław

Department of Chemistry, Warsaw University of Technology, ul. Noakowskiego 3, 00-664 Warsaw, Poland to form phenazines [7]. Other methods are the condensation of ortho-phenylenediamines with ortho-quinones (path d) [8], reaction of benzofuroxanes and phenols (the Beirut reaction, path e) [9], and palladium-catalyzed cyclization of 2-amino-2'-bromophenylenediamines (path f) [10].

The classic Wohl-Aue synthesis of phenazines consists in the reaction of anilines with nitroarenes under harsh basic conditions, usually by heating of both starting materials with sodium or potassium hydroxide at $200{ }^{\circ} \mathrm{C}$ [5]. In recent years we extensively studied nucleophilic aromatic substitution reactions of hydrogen in nitroarenes [11-15]. During these studies we have found that anilines react with nitrobenzene derivatives under mild conditions in the presence of $t$-BuOK in DMF at $-50{ }^{\circ} \mathrm{C}$ to form 2-nitrosodiphenylamines that in turn upon treatment with acetic acid cyclized to phenazines (Scheme2) [16, 17].

Other transformations of 2-nitrosodiphenylamines into heterocyclic systems developed by us include reactions with benzyl aryl sulfones to form 1,2-diarylbenzimidazoles [18] and cyclocondensation with functionalized alkyl acetates, such as malonates, phenyl- and phosphonyl-acetates, leading to 1 -arylquinoxalin-2(1H)-ones $[16,19]$.

1,2-Benzo- and 1,2-heteroaryl-fused phenazines are of interest owing to their potential biological activity, as intercalators [20, 21], and antimicrobial agents [22, 23]. Reports on the synthesis of pyrrolo[3,2-a]phenazines are scarce. 1-(2-Aminoethyl)pyrrolo[3,2-a]phenazine was formed from 1,2-phenylenediamine and the 4,5-indoloquinone arising from electrochemical oxidation of 5-hydroxytryptamine [24]. Dipyrrolo[3,2-a:3,2- $h$ ]phenazines were synthesized in the oxidative dimerization of 5-aminoindoles [25]. Some pyrrolo[3,2-a]phenazine-10carboxamides, obtained from 4-aminoindole and 2-iodo-3nitrobenzoic acid, were tested as cytotoxic agents [26]. 
Scheme 1

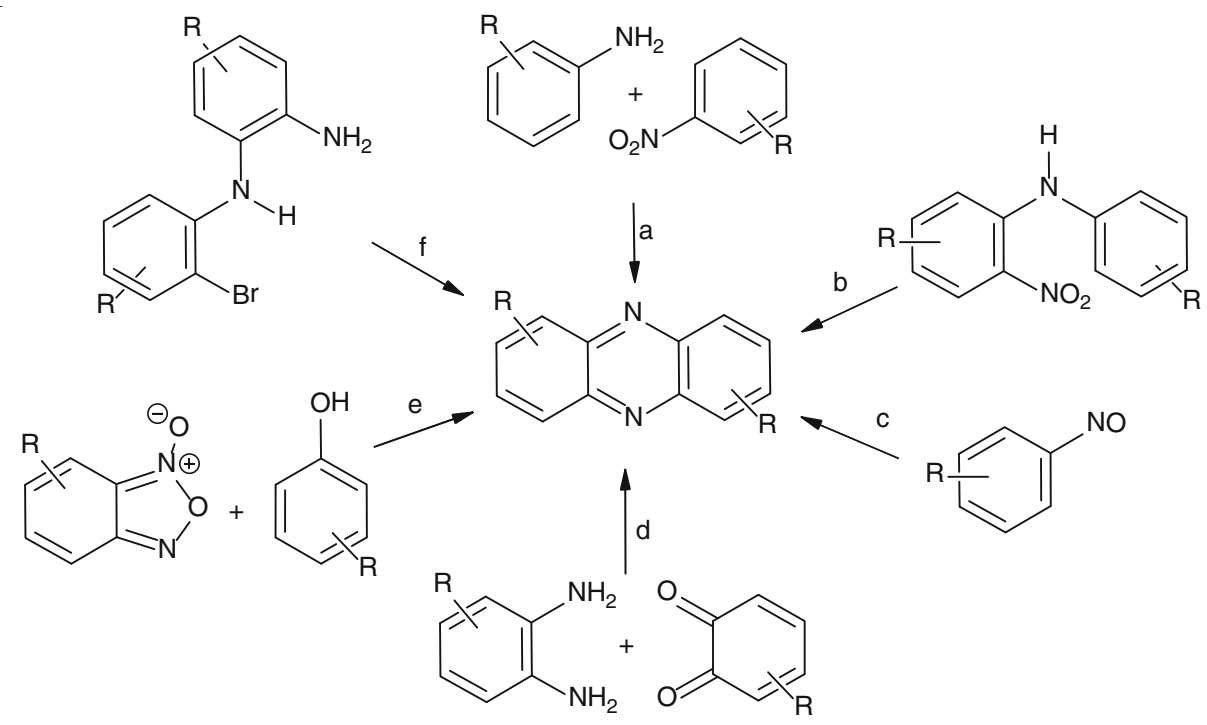

Scheme 2<smiles>[X]c1ccccc1N(Nc1ccccc1N=O)c1cccc([X])c1N=O</smiles>

\section{Results and discussion}

In this paper we present a simple synthesis of pyrrolo[3,2$a$ ]phenazines from nitroindoles and anilines. Thus when we treated 5-nitroindole derivatives $\mathbf{1}$ and anilines $\mathbf{2}$ with $t$ BuOK in DMF at $-50{ }^{\circ} \mathrm{C}$, the expected 4 - $(N$-arylamino $)$ 5-nitrosoindoles 3 were formed in good yields (Scheme 3 and Table 1).

Some of these compounds (3b and $\mathbf{3 f}$ ) proved unstable and thus after isolation without further purification they were used in the next step to form phenazines. The ${ }^{1} \mathrm{H}$ and ${ }^{13} \mathrm{C}$ NMR spectra of the obtained nitrosoamines 3 and 7 deserve some comments. In the spectra of some of these compounds we observed broadening of the signals corresponding to the protons and carbon atoms of the nitrososubstituted moiety and thus their full interpretation was troublesome. Such a signal broadening is probably due to a slow rotation of the nitroso group around the $\mathrm{C}-\mathrm{N}$ bond. A similar phenomenon was observed in the NMR spectra of 2-(alkylamino)- and 2-(arylamino)nitrosobenzenes [27, 28].
In our earlier papers we have shown that cyclization of $N$-(2-nitrosophenyl)anilines to phenazines proceeds satisfactorily in boiling acetic acid [16, 17], with $\mathrm{K}_{2} \mathrm{CO}_{3}$ in methanol at room temperature [17], or with $\mathrm{N}, \mathrm{O}$-bis(trimethylsilyl)acetamide (BSA) [17]. Attempted cyclization of the model nitroso compound $\mathbf{3 d}$ in boiling acetic acid was unsuccessful; the starting material was consumed within $90 \mathrm{~min}$ (TLC control) but no defined products were obtained. No reaction of $\mathbf{3 d}$ was observed in the presence of $\mathrm{K}_{2} \mathrm{CO}_{3}$ in methanol. The cyclization of $\mathbf{3 d}$ occurs satisfactorily in the presence of BSA in DMF at $80{ }^{\circ} \mathrm{C}$ giving the expected pyrrolophenazine $4 \mathbf{d}$ in good yield. These reaction conditions were adapted to reactions of other 4- $(\mathrm{N}$ arylamino)indoles 3 . The results are summarized in the Table 1.

Alternatively, the pyrrolo[2,3- $a$ ]phenazines can be obtained from aminoindoles and nitroarenes (Scheme 4). Thus, when we reacted 4-aminoindole $6 \mathbf{a}$ with 4-nitroanisole (5) under standard conditions $\left(t\right.$-BuOK/DMF, $\left.-50{ }^{\circ} \mathrm{C}\right)$ the expected nitrosoaniline $7 \mathbf{a}$ was formed. Since the amine 7 a proved unstable, it was without purification subjected to 
Scheme 3<smiles>[R]n1c(C)cc2cc([N+](=O)[O-])ccc21</smiles>

1<smiles>[X]c1ccc(N)cc1</smiles>

2<smiles>[X]c1ccc(Nc2c(O)ccc3c2cc(C)n3[R])cc1</smiles>

$3 a-3 f$<smiles>[X]c1ccc2nc3c(ccc4c3cc(C)n4[R])nc2c1</smiles>

Table 1 Synthesis of nitrosoindoles $\mathbf{3}$ and pyrrolo[3,2-a]phenazines 4

\begin{tabular}{lllll}
\hline & $\mathrm{R}$ & $\mathrm{X}$ & Yield of $\mathbf{3 / \%}$ & Yield of $\mathbf{4} / \%$ \\
\hline a & $\mathrm{Me}$ & $\mathrm{Cl}$ & 65 & 65 \\
$\mathbf{b}$ & $\mathrm{CH} \mathrm{H}_{2} \mathrm{Ph}$ & $\mathrm{Cl}$ & $36^{\mathrm{a}, \mathrm{b}}$ & 88 \\
c & $n-\mathrm{C}_{8} \mathrm{H}_{17}$ & $\mathrm{CH}_{3}$ & 30 & 88 \\
d & $n-\mathrm{C}_{8} \mathrm{H}_{17}$ & $\mathrm{Cl}$ & 58 & 80 \\
e & $n-\mathrm{C}_{8} \mathrm{H}_{17}$ & $\mathrm{OCH}_{3}$ & 50 & 71 \\
f & $n-\mathrm{C}_{8} \mathrm{H}_{17}$ & $\mathrm{CF}_{3}$ & $-{ }^{\text {b }}$ & 34 \\
\hline
\end{tabular}

a Yield of the crude product

b The crude product without purification was subjected to cyclization to phenazine

reaction with BSA and cyclized to 9-methoxypyrrolo[3,2a]phenazine $\mathbf{4 g}$ that was isolated in $90 \%$ yield. Similarly 5-aminoindole $\mathbf{6 b}$ and 4-nitroanisole formed the relatively stable nitroso derivative $\mathbf{7 b}$ that was isolated in $40 \%$ yield. Treatment of the compound $\mathbf{7 b}$ with BSA led to isomeric 8-methoxypyrrolo[3,2-a]phenazine $\mathbf{4 h}$ in $64 \%$ yield.

These reactions show the versatility of the proposed approach to pyrrolophenazines enabling the synthesis of derivatives bearing substituents in the desired position of the heterocyclic system, as exemplified by the synthesis of 8- and 9-methoxy derivatives $\mathbf{4 g}$ and $\mathbf{4 e}$ that can be obtained from different nitroarene-amine pairs, namely 5 -nitroindole and para-anisidine or 5-aminoindole (6b) and 4-nitroanisole (5).

In summary, a novel two-step approach to pyrrolophenazines starting from easily available nitroindoles and anilines was developed. In an alternative reaction sequence the pyrrolophenazines can be obtained from nitroarenes and aminoindoles. The simplicity of this approach makes it an interesting alternative to other procedures.

\section{Experimental}

All reactions were performed under argon atmosphere. ${ }^{1} \mathrm{H}$ and ${ }^{13} \mathrm{C}$ NMR spectra were recorded on Bruker $500 \mathrm{MHz}$ spectrometer $\left(500 \mathrm{MHz}\right.$ for ${ }^{1} \mathrm{H}$ and $125 \mathrm{MHz}$ for ${ }^{13} \mathrm{C}$ spectra). Chemical shifts $(\delta)$ are expressed in ppm referred to TMS, coupling constants in Hertz. Mass spectra (EI, $70 \mathrm{eV}$ ) were obtained on an AMD-604 spectrometer. ESI mass spectra were obtained on SYNAPT G2-S HDMS. Merck silica gel $60 \mathrm{~F}_{254}$ plates were used for TLC. Merck silica gel 60 (230-400 mesh) was used for flash column chromatography.

\section{Typical procedure for synthesis of compounds 3 and 7}

\section{$\mathrm{N}$-(4-Chlorophenyl)-1,2-dimethyl-5-nitroso-1H-indol-}

4-amine (3a, $\left.\mathrm{C}_{16} \mathrm{H}_{14} \mathrm{ClN}_{3} \mathrm{O}\right)$

4-Chloroaniline $(0.32 \mathrm{~g}, 2.5 \mathrm{mmol})$ in $2 \mathrm{~cm}^{3} \mathrm{DMF}$ was added to a solution of $0.67 \mathrm{~g} \mathrm{t}$-BuOK $(6 \mathrm{mmol})$ in $10 \mathrm{~cm}^{3}$ DMF cooled to $-50{ }^{\circ} \mathrm{C}$. After $5 \mathrm{~min}$ a solution of $0.38 \mathrm{~g}$ 1,2-dimethyl-5-nitroindole ( $2 \mathrm{mmol}$ ) in $3 \mathrm{~cm}^{3} \mathrm{DMF}$ was added. The reaction was stirred at -50 to $-40{ }^{\circ} \mathrm{C}$ until the starting indole disappeared (1-2 h, TLC control, $\mathrm{SiO}_{2}$, toluene/ethyl acetate 10:1). Then the reaction mixture was 


\section{Scheme 4}

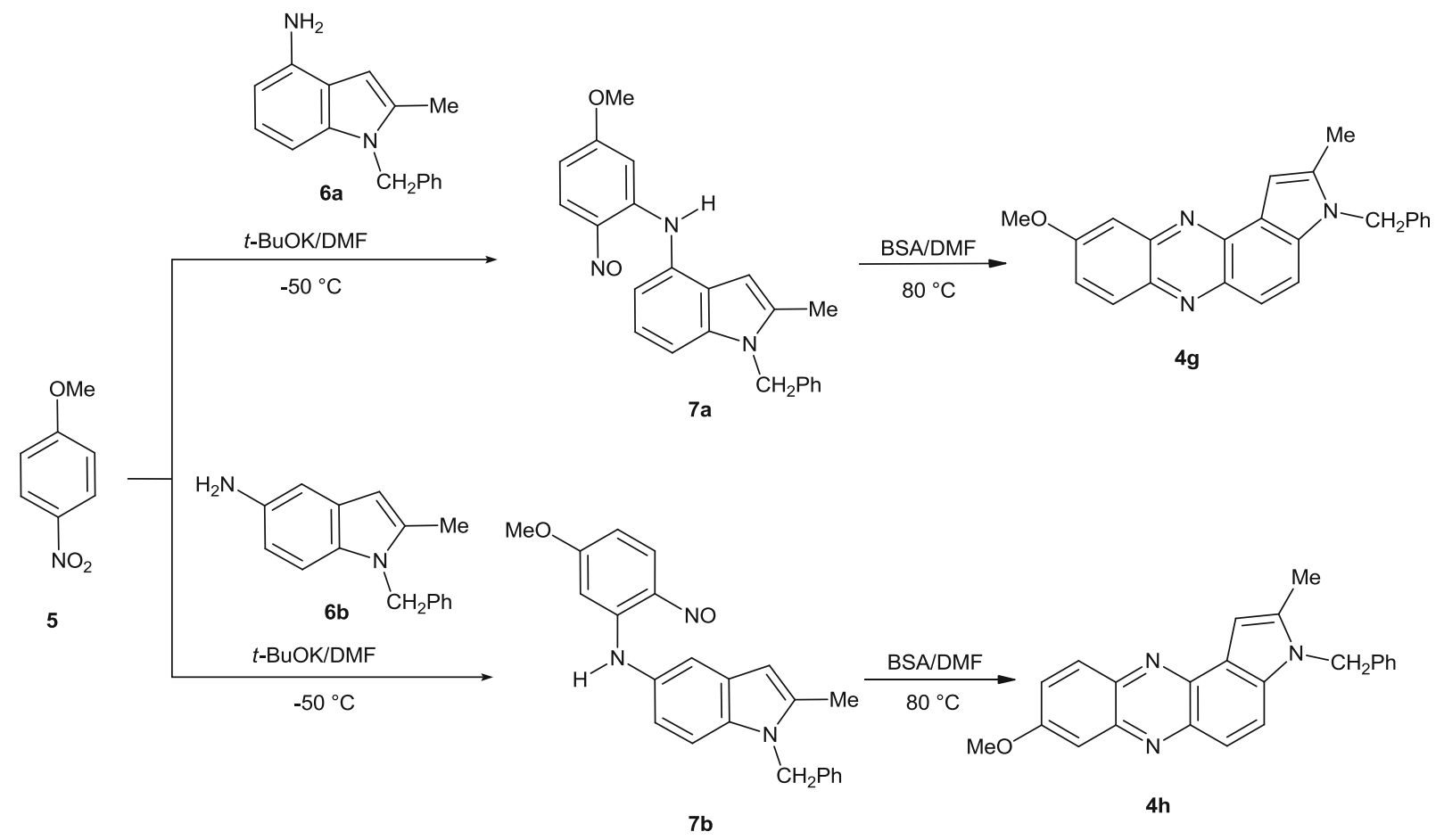

poured into $100 \mathrm{~cm}^{3}$ water with $5 \mathrm{~g} \mathrm{NH}_{4} \mathrm{Cl}$. The precipitate was dissolved in $100 \mathrm{~cm}^{3}$ EtOAc and dried with $\mathrm{Na}_{2} \mathrm{SO}_{4}$. After evaporation of solvent the product was purified by column chromatography $\left(\mathrm{SiO}_{2}\right.$, toluene/ethyl acetate). The product 3a was obtained as a dark red solid; m.p.: $>285{ }^{\circ} \mathrm{C}$ (decomp.); $R_{\mathrm{f}}=0.18$ (toluene/ethyl acetate 10:1); ${ }^{1} \mathrm{H}$ NMR (500 MHz, $\mathrm{CDCl}_{3}$ ): $\delta=2.21(\mathrm{~s}, 3 \mathrm{H}), 3.61$ (s, 3H), 5.41 (br s, 1H), 6.91 (br s, 1H), 7.15-7.26 (m, 2H), 7.37-7.38 (m, 2H), 8.14 (br s, $1 \mathrm{H}), 14.49$ (s, 1H) ppm; ${ }^{13} \mathrm{C}$ NMR (125 MHz, $\left.\mathrm{CDCl}_{3}\right): \delta=12.47,30.00,104.55$, $105.57,111.77,127.35,128.18,128.99,129.33,132.57$, 134.94, 137.38, 141.32, $153.62 \mathrm{ppm}$; MS (ESI): $\mathrm{m} / z=300$ $\left([\mathrm{M}+\mathrm{H}]^{+}, 100\right), 282$ (8); HRMS (ESI): calcd. for $\mathrm{C}_{16} \mathrm{H}_{15}^{35} \mathrm{ClN}_{3} \mathrm{O} 300.0904$, found 300.0905.

1-Benzyl-N-(4-chlorophenyl)-2-methyl-5-nitroso-1H-indol4-amine $\left(3 \mathrm{~b}, \mathrm{C}_{22} \mathrm{H}_{18} \mathrm{ClN}_{3} \mathrm{O}\right)$

Dark red unstable semisolid; MS (EI, $70 \mathrm{eV}$ ): $\mathrm{m} / \mathrm{z}=375$ $\left(\mathrm{M}^{+}, 42\right), 361$ (55), 358 (38), 344 (12), 323 (33), 267 (9), 253 (32), 235 (36), 219 (19), 91 (100); HRMS (ESI): calcd. for $\mathrm{C}_{22} \mathrm{H}_{18} \mathrm{ClN}_{3} \mathrm{NaO} 398.1031$, found 398.1040.

2-Methyl-N-(4-methylphenyl)-5-nitroso-1-octyl-1H-indol4-amine $\left(3 \mathrm{c}, \mathrm{C}_{24} \mathrm{H}_{31} \mathrm{~N}_{3} \mathrm{O}\right)$

Dark red oil; $R_{\mathrm{f}}=0.32$ (toluene/ethyl acetate $10: 1$ ); ${ }^{1} \mathrm{H}$ NMR $\left(500 \mathrm{MHz}, \mathrm{CDCl}_{3}\right): \delta=0.88(\mathrm{t}, J=7.2 \mathrm{~Hz}, 3 \mathrm{H})$, 1.26-1.32 (m, 10H), $1.70(\mathrm{~m}, 2 \mathrm{H}), 2.16(\mathrm{~s}, 3 \mathrm{H}), 2.42(\mathrm{~s}$, $3 \mathrm{H}), 3.93(\mathrm{t}, J=7.7 \mathrm{~Hz}, 2 \mathrm{H}), 6.60(\mathrm{~d}, J=8.4 \mathrm{~Hz}, 2 \mathrm{H})$, $6.96(\mathrm{~d}, J=8.4 \mathrm{~Hz}, 2 \mathrm{H}), 7.13-7.28(\mathrm{~m}, 3 \mathrm{H}), 14.69$ (br s, 1H) ppm; ${ }^{13} \mathrm{C}$ NMR $\left(125 \mathrm{MHz}, \mathrm{CDCl}_{3}\right): \delta=12.43,14.05$, $22.59,26.90,29.12,29.23,30.47,31.73,31.78,43.68$, $104.56,116.11,111.92,126.29,129.24,132.25,133.76$, 134.36, 135.78, 137.02, 140.54, 153.45 ppm; MS (ESI): $m / z=378\left(\mathrm{M}^{+}, 100\right)$; HRMS (ESI): calcd. for $\mathrm{C}_{24} \mathrm{H}_{32} \mathrm{~N}_{3} \mathrm{O}$ 378.2545 , found 378.2548 .

$\mathrm{N}$-(4-Chlorophenyl)-2-methyl-5-nitroso-1-octyl-1H-indol4-amine (3d, $\mathrm{C}_{23} \mathrm{H}_{28} \mathrm{ClN}_{3} \mathrm{O}$ )

Black solid; m.p.: $102-103{ }^{\circ} \mathrm{C} ; R_{\mathrm{f}}=0.40$ (toluene/ethyl acetate 10:1); ${ }^{1} \mathrm{H}$ NMR (500 $\left.\mathrm{MHz}, \mathrm{CDCl}_{3}\right): \delta=0.88(\mathrm{t}$, $J=7.1 \mathrm{~Hz}, 3 \mathrm{H}), 1.27-1.33(\mathrm{~m}, 10 \mathrm{H}), 1.70-1.73(\mathrm{~m}, 2 \mathrm{H})$, $2.20(\mathrm{~s}, 3 \mathrm{H}), 3.96(\mathrm{t}, J=7.5 \mathrm{~Hz}, 2 \mathrm{H}), 5.40$ (br s, 1H), 6.91 (br s, 1H), 7.24-7.29 m, 2H), 7.34-7.42 (m, 2H), 8.13 (br s, 1H), 14.54 (s, 1H) ppm; $\left.{ }^{13} \mathrm{C} \mathrm{NMR} \mathrm{(125} \mathrm{MHz,} \mathrm{CDCl}_{3}\right)$ : $\delta=12.44,14.02,22.55,26.86,29.09,29.19,30.43,31.70$, $43.76,104.95,105.90,121.14,127.41,128.91,129.38$, 132.29, 133.12, 134.46, 137.34, 140.80, 153.35 ppm; MS (ESI, $\mathrm{MeOH}): m / z=398\left([\mathrm{M}+\mathrm{H}]^{+}, 100\right), 380(10)$; HRMS (ESI): calcd. for $\mathrm{C}_{23} \mathrm{H}_{29}^{35} \mathrm{ClN}_{3} \mathrm{O} 398.1999$, found 398.1997.

$\mathrm{N}$-(4-Methoxyphenyl)-2-methyl-5-nitroso-1-octyl-1Hindol-4-amine $\left(3 \mathrm{e}, \mathrm{C}_{24} \mathrm{H}_{31} \mathrm{~N}_{3} \mathrm{O}_{2}\right)$

Black solid; m.p.: $77-79{ }^{\circ} \mathrm{C} ; \quad R_{\mathrm{f}}=0.24$ (toluene/ethyl acetate 10:1); ${ }^{1} \mathrm{H}$ NMR (500 $\left.\mathrm{MHz}, \mathrm{CDCl}_{3}\right): \delta=0.88(\mathrm{t}$, $J=7.1 \mathrm{~Hz}, 3 \mathrm{H}), 1.26-1.32(\mathrm{~m}, 10 \mathrm{H}), 1.67-1.71(\mathrm{~m}, 2 \mathrm{H})$, 
$2.16(\mathrm{~s}, 3 \mathrm{H}), 3.87(\mathrm{~s}, 3 \mathrm{H}), 3.94(\mathrm{t}, J=7.8,2 \mathrm{H}), 5.27(\mathrm{~s}$, $1 \mathrm{H}), 6.85(\mathrm{~d}, J=9.2 \mathrm{~Hz}, 1 \mathrm{H}), 6.94(\mathrm{~d}, J=8.7 \mathrm{~Hz}, 2 \mathrm{H})$, $7.20(\mathrm{~d}, J=8.7 \mathrm{~Hz}, 2 \mathrm{H}), 8.06(\mathrm{~d}, J=9.2 \mathrm{~Hz}, 1 \mathrm{H}), 14.63$ (s, 1H) ppm; ${ }^{13} \mathrm{C}$ NMR $\left(125 \mathrm{MHz}, \mathrm{CDCl}_{3}\right): \delta=12.39$, $14.03,22.56,26.87,29.10,29.20,30.44,31.71,43.67$, $55.50,104.53,106.00,111.83,114.42,127.81,131.10$, $132.15,133.77,135.00,140.50,153.28,158.66 \mathrm{ppm}$; MS (ESI, $\mathrm{MeOH}): m / z=394\left(\mathrm{M}^{+}, 100\right)$; HRMS (EI): calcd. for $\mathrm{C}_{24} \mathrm{H}_{32} \mathrm{~N}_{3} \mathrm{O}_{2}$ 394.2495, found 394.2494.

\section{1-Benzyl-N-(5-methoxy-2-nitrosophenyl)-2-methyl-1H- indol-4-amine (7a, $\mathrm{C}_{23} \mathrm{H}_{21} \mathrm{~N}_{3} \mathrm{O}_{2}$ )}

Dark red crystals; m.p.: $>115{ }^{\circ} \mathrm{C}$ (decomp); $R_{\mathrm{f}}=0.48$ (toluene/ethyl acetate 10:1); ${ }^{1} \mathrm{H}$ NMR (500 MHz, DMSO$\left.d_{6}\right): \delta=2.38(\mathrm{~s}, 3 \mathrm{H}), 3.75(\mathrm{~s}, 3 \mathrm{H}), 5.46(\mathrm{~s}, 2 \mathrm{H}), 6.23(\mathrm{~s}$, $1 \mathrm{H}), 6.43$ (br s, 1H), 6.68 (br s, 1H), 6.90-7.04 (m, 2H), $7.12(\mathrm{dd}, J=8.0,7.6 \mathrm{~Hz}, 1 \mathrm{H}), 7.18(\mathrm{~d}, J=7.6 \mathrm{~Hz}, 1 \mathrm{H})$, $7.21-7.25(\mathrm{~m}, \quad 1 \mathrm{H}), \quad 7.27-7.32 \quad(\mathrm{~m}, 2 \mathrm{H}), 7.36(\mathrm{~d}$, $J=8.0 \mathrm{~Hz}, 1 \mathrm{H}), 8.53$ (br s, $1 \mathrm{H}), 13.21$ (br s, $1 \mathrm{H}) \mathrm{ppm}$; ${ }^{13} \mathrm{C}$ NMR (125 MHz, DMSO- $\left.d_{6}\right): \delta=46.48,56.43$, 60.20 , 95.30, 98.02, 108.86, 109.98, 115.01, 121.44, $123.99,126.57,127.62,129.11,138.40,138.55 \mathrm{ppm}$ (spectrum not fully legible); MS (ESI, MeOH): $m / z=394\left([\mathrm{M}+\mathrm{Na}]^{+}\right), 372\left([\mathrm{M}+\mathrm{H}]^{+}\right) ;$HRMS (ESI, $\left.[\mathrm{M}+1]^{+}\right)$: calcd. for $\mathrm{C}_{23} \mathrm{H}_{22} \mathrm{~N}_{3} \mathrm{O}_{2}$ 372.1707, found 372.1718 .

\section{1-Benzyl-N-(5-methoxy-2-nitrosophenyl)-2-methyl-1H- indol-5-amine (7b, $\left.\mathrm{C}_{23} \mathrm{H}_{21} \mathrm{~N}_{3} \mathrm{O}_{2}\right)$}

Dark brown crystals; yield $40 \%$; m.p.: $>90{ }^{\circ} \mathrm{C}$ (decomp); $R_{\mathrm{f}}=0.38 \quad$ (toluene/ethyl acetate $\left.10: 1\right) ;{ }^{1} \mathrm{H} \quad \mathrm{NMR}$ $\left(500 \mathrm{MHz}, \mathrm{DMSO}-d_{6}\right): \delta=2.37(\mathrm{~s}, 3 \mathrm{H}), 3.73(\mathrm{~s}, 3 \mathrm{H})$, $5.44(\mathrm{~s}, 2 \mathrm{H}), 6.34(\mathrm{~s}, 1 \mathrm{H}), 6.40(\mathrm{br} \mathrm{s}, 1 \mathrm{H}), 6.64(\mathrm{br} \mathrm{s}, 1 \mathrm{H})$, 7.00-7.10 (m, 3H), 7.21-7.27 (m, 1H), 7.28-7.35 (m, 2H), 7.45 (d, $J=8.6 \mathrm{~Hz}, 1 \mathrm{H}), 7.50$ (br s, 1H), 12.98 (br s, 1H) ppm; ${ }^{13} \mathrm{C}$ NMR (125 MHz, DMSO- $\left.d_{6}\right): \delta=12.49,45.87$, $55.88,93.82,100.39,109.09,110.51,115.62,117.91$, $122.85,125.85,126.17,127.13,128.19,128.63,135.25$, 138.17, 138.46, 142.05, 153.48, 166.67 ppm; MS (ESI, $\mathrm{MeOH}): \quad m / z=394 \quad\left([\mathrm{M}+\mathrm{Na}]^{+}\right), \quad 372\left([\mathrm{M}+\mathrm{H}]^{+}\right)$; HRMS (ESI, $[\mathrm{M}+1]^{+}$): calcd. for $\mathrm{C}_{23} \mathrm{H}_{22} \mathrm{~N}_{3} \mathrm{O}_{2}$ 372.1707, found 372.1713 .

\section{Typical procedure for synthesis of compounds 4}

\section{8-Chloro-2,3-dimethylpyrrolo[3,2-a]phenazine}

\section{$\left(4 \mathbf{a}, \mathrm{C}_{16} \mathrm{H}_{12} \mathrm{ClN}_{3}\right)$}

To $200 \mathrm{mg}$ 4-arylamino-5-nitrosoindole $3(0.66 \mathrm{mmol})$ dissolved in $10 \mathrm{~cm}^{3}$ DMF was added $0.67 \mathrm{~g} \mathrm{~N}, O$-bis(trimethylsilyl)acetamide $(3.3 \mathrm{mmol})$. The reaction mixture was stirred at $80{ }^{\circ} \mathrm{C}$ for $12-24 \mathrm{~h}$ (TLC control, $n$-hexane/ ethyl acetate $4: 1)$. Then the reaction mixture was poured into $100 \mathrm{~cm}^{3}$ water. The product was separated, dissolved in $50 \mathrm{~cm}^{3}$ EtOAc, and dried with $\mathrm{Na}_{2} \mathrm{SO}_{4}$. After evaporation of the solvent the product was purified by column chromatography $\left(\mathrm{SiO}_{2}, n\right.$-hexane/ethyl acetate 4:1). Product 4a was obtained in the form of orange crystals; m.p.: $>300{ }^{\circ} \mathrm{C} ; R_{\mathrm{f}}=0.22(n$-hexane/ethyl acetate 4:1); ${ }^{1} \mathrm{H}$ NMR (500 MHz, DMF- $\left.d_{7}\right): \delta=2.59$ (s, 3H), 3.96 (s, 3H), $7.18(\mathrm{~s}, 1 \mathrm{H}), 7.76(\mathrm{~d}, J=9.4 \mathrm{~Hz}, 1 \mathrm{H}), 7.85(\mathrm{dd}$, $J=9.0,2.25 \mathrm{~Hz}, 1 \mathrm{H}), 8.18(\mathrm{~d}, J=9.4 \mathrm{~Hz}, 1 \mathrm{H}), 8.24-8.26$ $(\mathrm{m}, 2 \mathrm{H}) \mathrm{ppm} ;{ }^{13} \mathrm{C}$ NMR $\left(125 \mathrm{MHz}, \mathrm{DMF}-d_{7}\right): \delta=12.33$, $27.56,102.93,120.37,121.59,122.54,128.28,130.63$, $131.22,133.70,135.48,137.46,140.45,140.98,141.86$, $143.19 \mathrm{ppm}$; MS (EI, $70 \mathrm{eV}): \mathrm{m} / \mathrm{z}=281\left(\mathrm{M}^{+}, 100\right), 266$ (8); HRMS (EI): calcd. for $\mathrm{C}_{16} \mathrm{H}_{12} \mathrm{ClN}_{3} 281.0720$, found 281.0717.

\section{3-Benzyl-8-chloro-2-methylpyrrolo[3,2-a]phenazine $\left(4 \mathbf{b}, \mathrm{C}_{22} \mathrm{H}_{16} \mathrm{ClN}_{3}\right)$}

Yellow crystals; m.p.: $223-225{ }^{\circ} \mathrm{C} ; R_{\mathrm{f}}=0.37$ ( $n$-hexane/ ethyl acetate $4: 1) ;{ }^{1} \mathrm{H}$ NMR $\left(500 \mathrm{MHz}, \mathrm{CDCl}_{3}\right): \delta=2.50$ $(\mathrm{d}, J=0.8 \mathrm{~Hz}, 3 \mathrm{H}), 5.48(\mathrm{~s}, 2 \mathrm{H}), 6.9-7.00(\mathrm{~m}, 2 \mathrm{H})$, $7.26-7.32(\mathrm{~m}, 3 \mathrm{H}), 7.73(\mathrm{dd}, J=9.1,2.3 \mathrm{~Hz}, 1 \mathrm{H}), 7.77$ (d, $J=9.3 \mathrm{~Hz}, 1 \mathrm{H}), 7.79(\mathrm{~d}, J=9.3 \mathrm{~Hz}, 1 \mathrm{H}), 8.23(\mathrm{~d}$, $J=2.3 \mathrm{~Hz}, 1 \mathrm{H}), 8.26(\mathrm{~d}, J=9.2 \mathrm{~Hz}, 1 \mathrm{H}) \mathrm{ppm} ;{ }^{13} \mathrm{C}$ NMR $\left(125 \mathrm{MHz}, \mathrm{CDCl}_{3}\right): \delta=12.86,42.14,103.48$, $118.98,122.00,122.26,125.83,127.74,127.87,129.02$, $130.22,130.58,134.09,134.58,136.09,136.91,139.71$, $140.39,141.38,142.48 \mathrm{ppm} ; \mathrm{MS}$ (ESI): $\mathrm{m} / z=358$ $\left([\mathrm{M}+\mathrm{H}]^{+}\right) ; \quad$ HRMS (ESI): calcd. for $\mathrm{C}_{22} \mathrm{H}_{17} \mathrm{ClN}_{3}$ 358.1111 , found 358.1113 .

\section{2,8-Dimethyl-3-octylpyrrolo[3,2-a]phenazine}

$\left(4 \mathbf{c}, \mathrm{C}_{24} \mathrm{H}_{29} \mathrm{~N}_{3}\right)$

Brown-red solid; m.p.: $133-135{ }^{\circ} \mathrm{C} ; R_{\mathrm{f}}=0.54(n$-hexane/ ethyl acetate 4:1); ${ }^{1} \mathrm{H}$ NMR $\left(500 \mathrm{MHz}, \mathrm{CDCl}_{3}\right): \delta=0.87$ (br s, 3H), 1.15-1.45 (m, 10H), $1.82(\mathrm{~m}, 2 \mathrm{H}), 2.54(\mathrm{~s}, 3 \mathrm{H})$, $2.64(\mathrm{~s}, 3 \mathrm{H}), 4.18(\mathrm{~m}, 2 \mathrm{H}), 7.26(\mathrm{~s}, 1 \mathrm{H}), 7.63(\mathrm{br} \mathrm{d}$, $J=8.0 \mathrm{~Hz}, 1 \mathrm{H}), 7.75-7.87(\mathrm{~m}, 2 \mathrm{H}), 7.95-8.07(\mathrm{~m}, 1 \mathrm{H})$, 8.21 (br d, $J=8.0 \mathrm{~Hz}, 1 \mathrm{H}$ ) ppm; ${ }^{13} \mathrm{C}$ NMR $(125 \mathrm{MHz}$, $\left.\mathrm{CDCl}_{3}\right): \delta=12.84,14.01,21.99,22.56,26.96,29.13$, 29.26, 30.97, 31.71, 43.88, 102.79, 118.16, 121.41, 122.00, $127.54,128.38,132.19,133.81,135.17,138.76,139.23$, 140.52, 141.27, $141.77 \mathrm{ppm}$; MS (EI, $70 \mathrm{eV}): \mathrm{m} / \mathrm{z}=359$ $\left(\mathrm{M}^{+}, 100\right), 344$ (7), 316 (5), 288 (8), 274 (6), 260 (47), 246 (27), 233 (99); HRMS (EI): calcd. for $\mathrm{C}_{24} \mathrm{H}_{29} \mathrm{~N}_{3} 359.2361$, found 359.2357 .

\section{8-Chloro-2-methyl-3-octylpyrrolo[3,2-a]phenazine}

$\left(4 d, \mathrm{C}_{23} \mathrm{H}_{26} \mathrm{ClN}_{3}\right.$ )

Yellow crystals; m.p.: $157-159{ }^{\circ} \mathrm{C} ; R_{\mathrm{f}}=0.70$ ( $n$-hexane/ ethyl acetate 4:1); ${ }^{1} \mathrm{H}$ NMR (500 $\left.\mathrm{MHz}, \mathrm{CDCl}_{3}\right): \delta=0.86$ $(\mathrm{t}, J=7.1 \mathrm{~Hz}, 3 \mathrm{H}), 1.26-1.40(\mathrm{~m}, 10 \mathrm{H}), 1.79-1.85(\mathrm{~m}$, 2H), 2.55 (s, 3H), 4.19 (t, $J=7.6 \mathrm{~Hz}, 2 \mathrm{H}), 7.25(\mathrm{~s}, 1 \mathrm{H})$, $7.71(\mathrm{dd}, J=9.1,2.2 \mathrm{~Hz}, 1 \mathrm{H}), 7.78(\mathrm{~d}, J=9.3 \mathrm{~Hz}, 1 \mathrm{H})$, $7.84(\mathrm{~d}, J=9.3 \mathrm{~Hz}, 1 \mathrm{H}), 8.23-8.25(\mathrm{~m}, 2 \mathrm{H}) \mathrm{ppm} ;{ }^{13} \mathrm{C}$ NMR (125 MHz, $\left.\mathrm{CDCl}_{3}\right): \delta=12.84,14.01,22.56,26.95$, 
$29.12,29.25,30.98,31.71,43.95,103.11,119.05,121.41$, $121.88,127.79,130.10,130.46,133.94,134.06,135.54$, 139.66, 140.16, 141.20, 142.39 ppm; MS (EI, $70 \mathrm{eV})$ : $m / z=379\left(\mathrm{M}^{+}, 100\right), 282$ (19), 281 (15), 266 (23); HRMS (EI): calcd. for $\mathrm{C}_{23} \mathrm{H}_{26}^{35} \mathrm{ClN}_{3} 379.1815$, found 379.1818.

\section{8-Methoxy-2-methyl-3-octylpyrrolo[3,2-a]phenazine \\ $\left(4 \mathrm{e}, \mathrm{C}_{24} \mathrm{H}_{29} \mathrm{~N}_{3} \mathrm{O}\right)$}

Yellow crystals; m.p.: $122-124{ }^{\circ} \mathrm{C} ; R_{\mathrm{f}}=0.38$ ( $n$-hexane/ ethyl acetate 4:1); ${ }^{1} \mathrm{H}$ NMR (500 MHz, $\mathrm{CDCl}_{3}$ ): $\delta=0.86$ $(\mathrm{t}, J=7.1 \mathrm{~Hz}, 3 \mathrm{H}), 1.25-1.39(\mathrm{~m}, 10 \mathrm{H}), 1.81(\mathrm{~m}, 2 \mathrm{H})$, $2.56(\mathrm{~s}, 3 \mathrm{H}), 4.01(\mathrm{~s}, 3 \mathrm{H}), 4.19(\mathrm{t}, J=7.6 \mathrm{~Hz}, 2 \mathrm{H}), 7.22$ (s, $1 \mathrm{H}), 7.46-7.47(\mathrm{~m}, 2 \mathrm{H}), 7.77(\mathrm{~d}, J=9.2 \mathrm{~Hz}, 1 \mathrm{H}), 7.81(\mathrm{~d}$, $J=9.2 \mathrm{~Hz}, 1 \mathrm{H}), 8.16(\mathrm{~m}, 1 \mathrm{H}) \mathrm{ppm} ;{ }^{13} \mathrm{C}$ NMR $(125 \mathrm{MHz}$, $\left.\mathrm{CDCl}_{3}\right): \delta=12.86,14.02,22.56,26.96,29.13,29.27$, $30.95,31.71,43.83,55.70,102.25,105.11,117.89,120.99$, $122.43,124.19,130.07,133.44,135.15,138.29,138.89$, 141.66, 142.74, $159.68 \mathrm{ppm}$; MS (EI, $70 \mathrm{eV}): \mathrm{m} / z=375$ ( $\left.\mathrm{M}^{+}, 100\right), 276$ (21), 262 (12), 233 (20), 219 (10); HRMS (EI): calcd. for $\mathrm{C}_{24} \mathrm{H}_{29} \mathrm{~N}_{3} \mathrm{O}$ 375.2311, found 375.2325.

\section{2-Methyl-3-octyl-8-(trifluoromethyl)pyrrolo[3,2-a]phen-}

azine (4f, $\left.\mathrm{C}_{24} \mathrm{H}_{26} \mathrm{~F}_{3} \mathrm{~N}_{3}\right)$

Orange crystals; m.p.: $127-129{ }^{\circ} \mathrm{C} ; R_{\mathrm{f}}=0.74$ ( $n$-hexane/ ethyl acetate 4:1); ${ }^{1} \mathrm{H}$ NMR (500 $\left.\mathrm{MHz}, \mathrm{CDCl}_{3}\right): \delta=0.87$ $(\mathrm{t}, J=7.1 \mathrm{~Hz}, 3 \mathrm{H}), 1.26-1.40(\mathrm{~m}, 10 \mathrm{H}), 1.84(\mathrm{~m}, 2 \mathrm{H})$, $2.57(\mathrm{~s}, 3 \mathrm{H}), 4.22(\mathrm{t}, J=7.6 \mathrm{~Hz}, 2 \mathrm{H}), 7.29(\mathrm{~s}, 1 \mathrm{H}), 7.82(\mathrm{~d}$, $J=9.3 \mathrm{~Hz}, 1 \mathrm{H}), 7.88(\mathrm{~d}, J=9.3 \mathrm{~Hz}, 1 \mathrm{H}), 7.93(\mathrm{dd}$, $J=9.0,2.0 \mathrm{~Hz}, 1 \mathrm{H}), 8.41(\mathrm{~d}, J=9.0 \mathrm{~Hz}, 1 \mathrm{H}), 8.58(\mathrm{~s}$, 1H) ppm; ${ }^{13} \mathrm{C}$ NMR $\left(125 \mathrm{MHz}, \mathrm{CDCl}_{3}\right): \delta=12.87,14.03$, $22.57,26.96,29.13,29.26,31.02,31.72,44.03,103.46$, $119.40,121.70,121.75,124.02$ (q, $J=272 \mathrm{~Hz}), 124.49$, $127.67(\mathrm{q}, J=4.9 \mathrm{~Hz}), 129.61(\mathrm{q}, J=32 \mathrm{~Hz}), 130.18$, 134.47, 135.72, 139.79, 140.77, 142.42, 143.05 ppm; MS (EI, $70 \mathrm{eV}): \mathrm{m} / \mathrm{z}=413\left(\mathrm{M}^{+}, 100\right), 315$ (45), 301 (11), 300 (23), 287 (9); HRMS (EI): calcd. for $\mathrm{C}_{24} \mathrm{H}_{26} \mathrm{~F}_{3} \mathrm{~N}_{3}$ 413.2079, found 413.2090.

\section{3-Benzyl-9-methoxy-2-methylpyrrolo[3,2-a]phenazine $\left(4 \mathrm{~g}, \mathrm{C}_{23} \mathrm{H}_{19} \mathrm{~N}_{3} \mathrm{O}\right)$}

Yield $90 \%$; orange crystals; m.p.: $>250{ }^{\circ} \mathrm{C} ; R_{\mathrm{f}}=0.18(n-$ hexane/ethyl acetate $4: 1) ;{ }^{1} \mathrm{H}$ NMR (500 MHz, DMSO- $\left.d_{6}\right)$ : $\delta=2.48(\mathrm{~s}, 3 \mathrm{H}), 4.03(\mathrm{~s}, 3 \mathrm{H}), 5.65(\mathrm{~s}, 2 \mathrm{H}), 7.04-7.08(\mathrm{~m}$, $2 \mathrm{H}), 7.18(\mathrm{~s}, 1 \mathrm{H}), 7.23-7.35(\mathrm{~m}, 3 \mathrm{H}), 7.52(\mathrm{dd}, J=9.3$, $2.5 \mathrm{~Hz}, 1 \mathrm{H}), 7.57 \quad(\mathrm{~d}, \quad J=2.5 \mathrm{~Hz}, 1 \mathrm{H}), 7.72 \quad(\mathrm{~d}$, $J=9.0 \mathrm{~Hz}, 1 \mathrm{H}), 8.08(\mathrm{~d}, J=9.3 \mathrm{~Hz}, 1 \mathrm{H}), 8.10(\mathrm{~d}$, $J=9.0 \mathrm{~Hz}, 1 \mathrm{H}) \mathrm{ppm} ;{ }^{13} \mathrm{C}$ NMR $\left(125 \mathrm{MHz}\right.$, DMSO- $\left.d_{6}\right)$ : $\delta=13.05,46.82,56.37,102.99,105.45,118.18,121.75$, $121.94,123.76,126.66,127.77,129.22,130.80,135.02$, $136.36, \quad 138.05,138.43,139.43,140.03,143.53$, $160.72 \mathrm{ppm}$; MS (ESI): $m / z=354\left([\mathrm{M}+\mathrm{H}]^{+}\right)$; HRMS (ESI): calcd. for $\mathrm{C}_{23} \mathrm{H}_{20} \mathrm{~N}_{3} \mathrm{O}$ 354.1601, found 354.1615.
3-Benzyl-8-methoxy-2-methylpyrrolo[3,2-a]phenazine

$\left(\mathbf{4 h}, \mathrm{C}_{23} \mathrm{H}_{19} \mathrm{~N}_{3} \mathrm{O}\right)$

Yield $64 \%$; yellow crystals; m.p.: $225-227{ }^{\circ} \mathrm{C} ; R_{\mathrm{f}}=0.22$ (n-hexane/ethyl acetate 4:1); ${ }^{1} \mathrm{H}$ NMR $\left(500 \mathrm{MHz}, \mathrm{CDCl}_{3}\right)$ : $\delta=2.48(\mathrm{~d}, J=0.7 \mathrm{~Hz}, 3 \mathrm{H}), 4.01(\mathrm{~s}, 3 \mathrm{H}), 5.45(\mathrm{~s}, 2 \mathrm{H})$, 6.97 (br s, 1H), 7.22-7.30 (m, 3H), 7.32 (s, 1H), 7.45-7.50 $(\mathrm{m}, 2 \mathrm{H}), 7.73(\mathrm{~d}, J=9.3 \mathrm{~Hz}, 1 \mathrm{H}), 7.75(\mathrm{~d}, J=9.3 \mathrm{~Hz}$, $1 \mathrm{H}), 8.18(\mathrm{~d}, J=9.0 \mathrm{~Hz}, 1 \mathrm{H}) \mathrm{ppm} ;{ }^{13} \mathrm{C}$ NMR $(125 \mathrm{MHz}$, $\left.\mathrm{CDCl}_{3}\right): \delta=12.83,47.00,55.73,102.75,105.06,117.86$, $121.47,122.73,124.38,125.83,127.59,128.93,130.09$, $133.98,135.72,137.17,138.20,138.98,141.65,142.82$, $159.08 \mathrm{ppm}$; MS (ESI): $m / z=354\left([\mathrm{M}+\mathrm{H}]^{+}\right)$; HRMS (ESI): calcd. for $\mathrm{C}_{23} \mathrm{H}_{20} \mathrm{~N}_{3} \mathrm{O}$ 354.1601, found 354.1604.

Acknowledgments This research was supported by the National Scientific Center, Grant NN 204193038.

Open Access This article is distributed under the terms of the Creative Commons Attribution License which permits any use, distribution, and reproduction in any medium, provided the original author(s) and the source are credited.

\section{References}

1. Laursen JB, Nielsen J (2004) Chem Rev 104:1663

2. Beifuss U, Tietze M (2005) Top Curr Chem 244:77

3. Mentel M, Ahuja EG, Mavrodi DV, Breinbauer R, Thomashow LS, Blankenfeldt W (2009) ChemBioChem 10:2295

4. Mavrodi DV, Blankenfeldt W, Thomashow LS (2006) Annu Rev Phytopathol 44:417

5. Wohl A, Aue W (1901) Chem Ber 34:2442

6. Gaertner G, Holliman FG, Gray A (1962) Tetrahedron 18:1105

7. Bamberger E, Ham W (1911) Liebigs Ann Chem 382:82

8. Kehrmann F, Mermod C (1927) Helv Chim Acta 10:62

9. Haddadin MJ, Issidorides CH (1993) Heterocycles 35:1503

10. Emoto T, Kubosaki N, Yamagiwa Y, Kamikawa T (2000) Tetrahedron Lett 41:355

11. Mąkosza M (2011) Synthesis 2341

12. Mąkosza M, Wojciechowski K (2011) Nucleophilic substitution of hydrogen-an efficient tool in synthesis of heterocyclic compounds. In: Attanasi $\mathrm{O}$ (ed) Targets in heterocyclic systems: chemistry and properties, vol 14 . Societa Chimica Italiana, Rome, p 19

13. Mąkosza M (2010) Chem Soc Rev 39:2855

14. Mąkosza M, Wojciechowski K (2004) Chem Rev 104:2631

15. Mąkosza M, Wojciechowski K (2001) Heterocycles 54:445

16. Wróbel Z, Kwast A (2007) Synlett 1525

17. Kwast A, Stachowska K, Trawczyński A, Wróbel Z (2011) Tetrahedron Lett 52:6484

18. Wróbel Z, Stachowska K, Grudzień K, Kwast A (2011) Synlett 1439

19. Wróbel Z, Stachowska K, Kwast A, Gościk A, Królikiewicz M, Pawłowski R, Turska I (2013) Helv Chim Acta 96:956

20. Cimmino A, Evidente A, Mathieu V, Andolfi A, Lefranc F, Kornienko A, Kiss R (2012) Nat Prod Rep 29:487

21. Vicker N, Burgess L, Chuckowree IS, Dodd R, Folkes AJ, Hardick DJ, Hancox TC, Miller W, Milton J, Sohal S, Wang S, Wren 
SP, Charlton PA, Dangerfield W, Liddle C, Mistry P, Stewart AJ, Denny WA (2002) J Med Chem 45:721

22. Hussain H, Specht S, Sarite SR, Saeftel M, Hoerauf A, Schulz B, Krohn K (2011) J Med Chem 54:4913

23. Saleh O, Flinspach K, Westrich L, Kulik A, Gust B, Fiedler H-P, Heide L (2012) Beilstein J Org Chem 8:501

24. Wrona MZ, Dryhurst G (1987) J Org Chem 52:2817

25. Meesala R, Nagarajan R (2010) Synlett 2808
26. Gamage SA, Spicer JA, Rewcastle GW, Milton J, Sohal S, Dangerfield W, Mistry P, Vicker N, Charlton PA, Denny WA (2002) J Med Chem 45:740

27. Lipilin DL, Churakov AM, Ioffe SL, Strelenko YA, Tartakovsky VA (1999) Eur J Org Chem 29

28. Wirth S, Wallek AU, Zernickel A, Feil F, Sztiller-Sikorska M, Lesiak-Mieczkowska K, Bräuchle C, Lorenz I-P, Czyz M (2010) J Inorg Biochem 104:774 